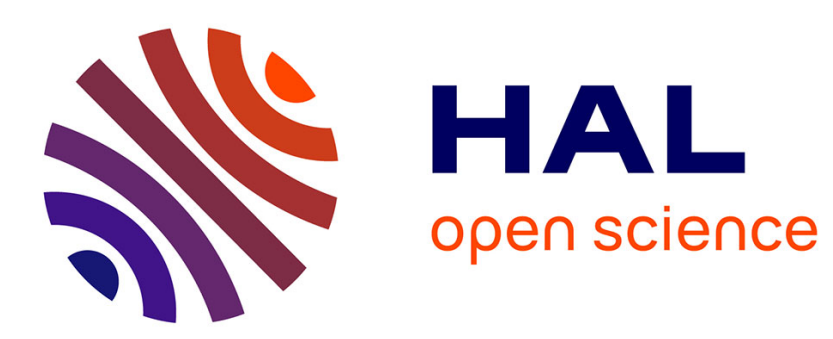

\title{
The Smurf transition: new insights on ageing from end-of-life studies in animal models
}

\author{
Michael Rera, Céline Vallot, Christel Lefrançois
}

\section{To cite this version:}

Michael Rera, Céline Vallot, Christel Lefrançois. The Smurf transition: new insights on ageing from end-of-life studies in animal models. Current Opinion in Oncology, 2018, 30 (1), pp.38-44. 10.1097/cco.0000000000000419 . hal-01981182

HAL Id: hal-01981182

https://hal-univ-rochelle.archives-ouvertes.fr/hal-01981182

Submitted on 3 May 2019

HAL is a multi-disciplinary open access archive for the deposit and dissemination of scientific research documents, whether they are published or not. The documents may come from teaching and research institutions in France or abroad, or from public or private research centers.
L'archive ouverte pluridisciplinaire HAL, est destinée au dépôt et à la diffusion de documents scientifiques de niveau recherche, publiés ou non, émanant des établissements d'enseignement et de recherche français ou étrangers, des laboratoires publics ou privés. 


\title{
The Smurf transition: new insights on ageing from end-of-life studies in animal models
}

\author{
Michael Rera ${ }^{\mathrm{a}}$. Céline Vallot ${ }^{\mathrm{b}}$, and Christel Lefrancois ${ }^{\mathrm{c}}$
}

\begin{abstract}
Purpose of review
Over the past 5 years, many articles were published concerning the prediction of high risk of mortality in apparently healthy adults, echoing the first description in 2011 of the Smurf phenotype, a harbinger of natural death in drosophila.
\end{abstract}

Recent findings

These recent findings suggest that the end-of-life is molecularly and physiologically highly stereotyped, evolutionarily conserved and predictable.

\begin{abstract}
Summary
Taken altogether, these results from independent teams using multiple organisms including humans draw the lines of future directions in ageing research. The ability to identify and study individuals about to die of natural causes with no apparent diseases is a game-changer in this field. In addition, the public health applications are potentially of tremendous impact in our ageing societies and raise important ethical questions.
\end{abstract}

\section{Keywords}

2PAC, ageing, death prediction, end-of-life, Smurfs

\section{INTRODUCTION}

Ageing is a major source of concern for human beings. We all undergo its damaging processes, yet it does not affect all of us equally. In a similar way, although several organisms are affected by ageing, not all organisms age the same way [1"]. We first need to define ageing to understand its nature. A commonly accepted definition of ageing describes it as a progressive worsening of organismal function leading to increasing age-specific mortality [2]. The success of this definition probably comes from the fact that we all, as human beings, observe an apparently progressive decay of our cognitive and physical capabilities as well as many of our visible features. Although this definition is still strongly rooted in the mind of gerontoscientists as well as the general public, recent data suggest that a more dramatic transition might occur during ageing. With the ability to detect such a transition comes the possibility to divide a population into its component subpopulations characterized by different instant mortality risks. With the identification of highly stereotyped physiological and/or molecular signatures comes the notion that end-of-life may be programmed and therefore predictable. These recent changes strongly affect the way we consider and study ageing. This review aims at highlighting these changes.

\section{Defining ageing influences how we study it}

As humans, we have built our understanding of ageing on simple day-to-day observations. We see an apparently progressive decrease of our own intellectual and physical capabilities, a progressive greying of our hair and wrinkles lining our faces. As such, the way ageing is described in the literature mostly refers to it as a continuous and progressive phenomenon. In their 2000 article entitled 'Why do we age?", Kirkwood and Austad [2] define it as "a

\footnotetext{
aUnité de Biologie Fonctionnelle et Adaptative (BFA) UMR8251 -

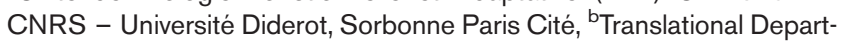
ment 'Dynamics of epigenetic alterations in breast cancer' UMR3244, Institut Curie, Paris and 'LIENSs UMR7266, CNRS-Université La Rochelle, La Rochelle, France

Correspondence to Michael Rera, Unité de Biologie Fonctionnelle et Adaptative (BFA) UMR8251 - CNRS - Université Diderot, Sorbonne Paris Cité, 4 Rue Marie Andrée Lagroua Weill Hallé, 75013 Paris, France. Tel: +33 1572779 52; e-mail: michael.rera@univ-paris-diderot.fr
}

Curr Opin Oncol 2017, 29:000-000

DOI:10.1097/CCO.0000000000000419 


\section{KEY POINTS}

- Ageing in two consecutive and distinct phases is evolutionarily conserved.

- Known hallmarks of ageing are not progressively affected by age but dramatically as individuals enter phase 2 of life.

- The phase 2 of life is molecularly and physiologically stereotyped.

- The recent literature describes multiple predictors of 5year all-causes mortality in humans.

- Studying human ageing using the 2-phase model of ageing would allow a better understanding of frailty in humans.

progressive worsening of organismal function leading to increasing age-specific mortality'. According to this definition, the general approach for studying ageing involves comparing groups of individuals from different chronological ages. Individuals of a same chronological age are then considered all equivalent (biological replicates). This approach for studying ageing has led to the description of hallmarks of ageing progressively changing with chronological age [3-8]. Although we cannot exclude that some changes occur progressively during ageing, all individuals of the same chronological age are not equivalent in terms of physiological age. This leads to significant changes of the ageing paradigm and to the way we study ageing.

\section{Ageing as a two-phase process in model organisms}

Instead of being a continuous decrease of organisms' capacity to cope with environmental challenges, recent findings suggest that ageing appears to occur through at least two distinct and consecutive phases separated by a dramatic transition. A phenomenon named 'spiral of death' was described in 2009 using drosophila [9]. This initial study predicted incoming mortality in female flies using fertility measurements, with a sharp decrease in fertility prior to death as opposed to a progressive decrease. The same authors later slightly elaborated on this phenomenon [10].

In 2011, a new age-related physiological phenotype was described for the first time, allowing for the identification of two subpopulations of Drosophila melanogaster of both sexes at any time point during the ageing process [11]. The phenotype is an in-vivo permeabilization of the intestine to a nontoxic blue food dye. Because the individuals become fully blue, I dubbed this phenotype 'Smurf'. In 2012, we showed that Smurf individuals were the only showing a high level of inflammation, loss of energy stores, low spontaneous mobility as well as a high risk of impending death [12]. We recently discovered that both male and female Smurfs show a dramatic decrease in fertility (Fig. 1). Prior to this seminal work, inflammation [13], energy stores and mobility [14] were described as being progressively and continuously affected by age. More strikingly, the Smurf phenotype is a strong predictor of impending death independently of the chronological age of individuals $\left[12,15^{-"}\right]$. We proposed a model of ageing in which the latter is a process consisting of at least two consecutive and distinct phases. Among these phases, only phase 2 is associated with the so-called 'age-related phenotypes' of which impending death is probably the most striking. Not only is this new theoretical framework supported by experimental data, but also by a mathematical model (dubbed the '2PAC' model) which describes longevity curves and allows for the reinterpretation of previously published ageing data such as gene expression analysis [15"'].

Furthermore, in 2016, we extended this 2-phase ageing model to other model organisms including different drosophila species, Caenorhabditis elegans,

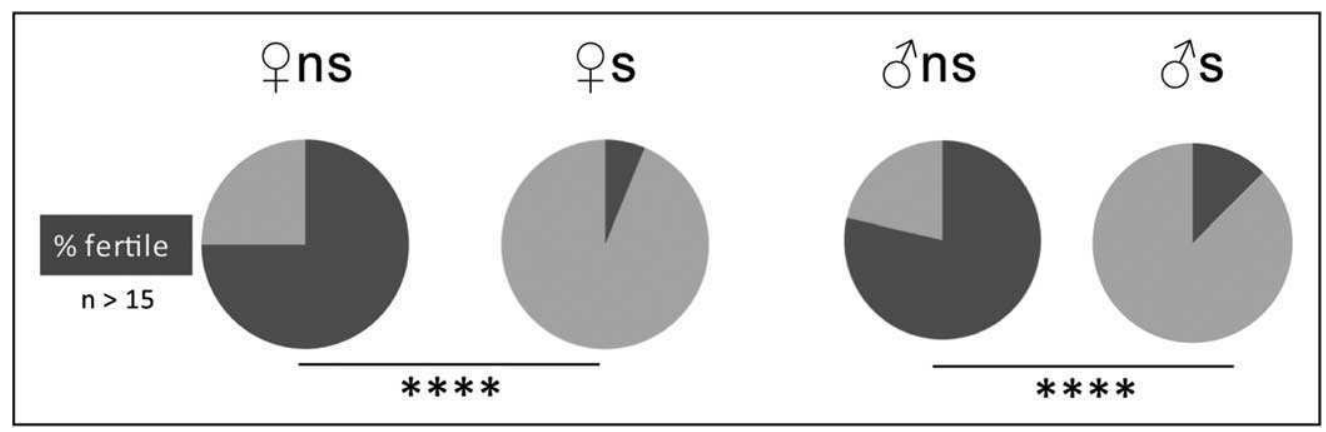

FIGURE 1. Mid-life fertility of both males and females either Smurf or not. Non-Smurf individuals (ns) are more than $75 \%$ fertile when only less than $9 \%$ of Smurf (s) individuals are. The samples were compared using a binomial test as the independent flies showed binary outcomes (fertile/nonfertile). ${ }^{* * *}$ represents a $P$-value $<5.10^{-7}$. 


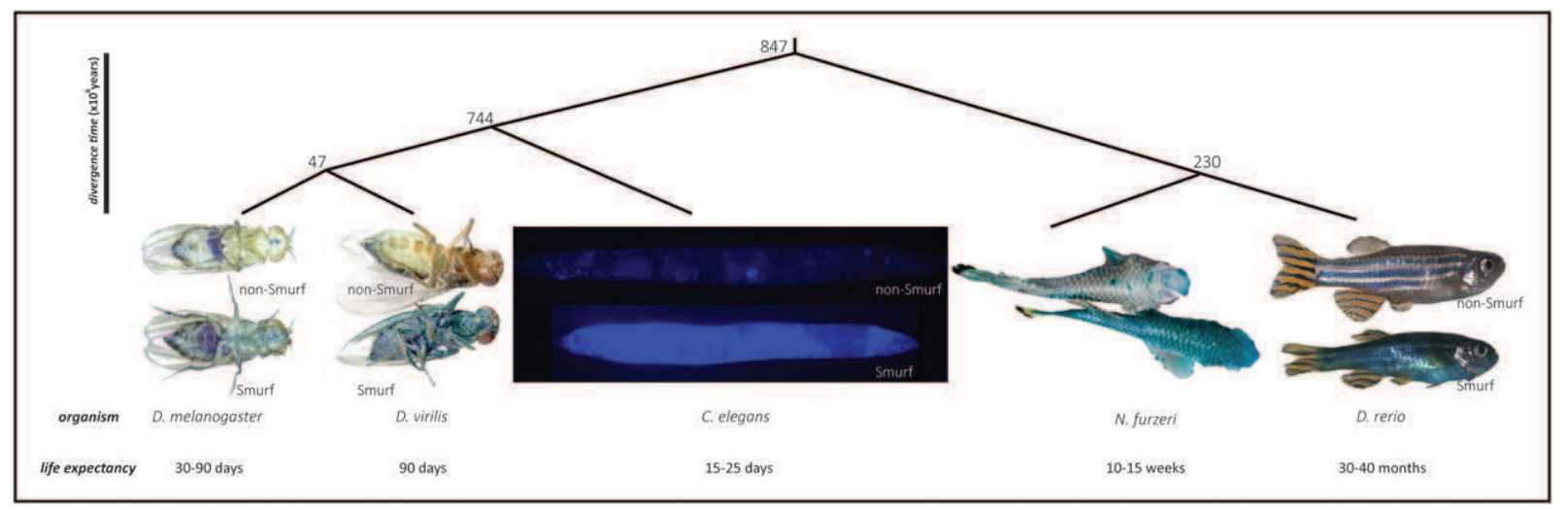

FIGURE 2. Evolutionary conservation of age-related intestinal permeability. From invertebrate to vertebrate model organisms, we showed an increasing proportion of individuals with an increased intestinal to the food dye blue \#1. In addition, we showed that this phenotype is a harbinger of death in multiple drosophila species, in nematodes as well as the zebrafish. (with permission from [16] under the CCBY licence).

Nothobranchius furzeri (unpublished preliminary work with Dr Christel Lefrançois) and Danio rerio [16"'] (adapted Fig. 2). At the exception of N. furzeri, we demonstrated that these organisms show the three most striking Smurf properties:

(1) an age-dependent linear increase in individuals' risk of intestinal 'leakage' (Smurfness)

(2) Smurfness is a harbinger of death

(3) Smurfness duration is mostly uncorrelated with chronological age

This study, showing a broad evolutionary conservation of a 2-phase ageing model, echoes older studies that suggest an age-dependent increase of intestinal permeability in baboons [17], rats [18] and in critically ill humans [19].

\section{Predicting high risk of impending death in ageing research}

Defining ageing not as one continuous phenomenon but as a 2-phase process allows us to develop new original approaches for studying ageing.

Firstly, the 2PAC model we proposed [15"'] to study longevity curves replaces the classical Gompertz and Weibull models parameters that are difficult to interpret [20-22] by three easily interpretable parameters that can be estimated experimentally:

(1) $a$ : the rate of increasing risk for transition from phase 1 to phase 2 (Smurf individuals)

(2) $b$ : the tolerance of the organisms to the Smurf transition

(3) $k$ : the mortality rate per se in phase 2

Thanks to this model, it is now possible to measure experimentally which parameter of ageing is affected by a given genetic/pharmacologic/environmental intervention modifying longevity. Interestingly, it is possible to use this model to reanalyze previously published data by assuming that the $k$ parameter is equal to previously published estimates for drosophila from different genetic backgrounds [15"'], or by scaling it up for other organisms assuming it is constant throughout life.

Secondly, as Smurf individuals are in a predeath phase of life that is physiologically stereotyped, individuals in this phase would hypothetically show a characteristic 'end-of-life transcriptional signature'. We identified such a signature thanks to an RNA sequencing of individuals from different chronological ages, either in the Smurf phase or not. The preliminary result obtained by principal component analysis shows that the Smurf state constitutes a large part of the effect observed in RNA samples, this effect being stronger than that of chronological age (Fig. 3a, b). It is significant that five hours after the Smurf transition, individuals have already acquired the transcriptional identity of a Smurf. This suggests that we can identify genes specifically associated with the high risk of impending death showed by individuals in this 'end-of-life phase', as well as genes whose expressions are modified prior to that transition. This study, currently in progress in the laboratory, also implies that at least the predeath part of the ageing process is molecularly stereotyped, if not programmed. Interestingly, the absolute number of genes differentially expressed in Smurfs and nonSmurfs decreases with chronological age. This suggests that the older individuals get, the closer they are to entering phase 2 (Fig. 3b, c). It is a molecular equivalent of the chronological age-dependent increase of an individual's probability to become a Smurf. Whether these changes can predict the entry in the Smurf phase is under investigation. 


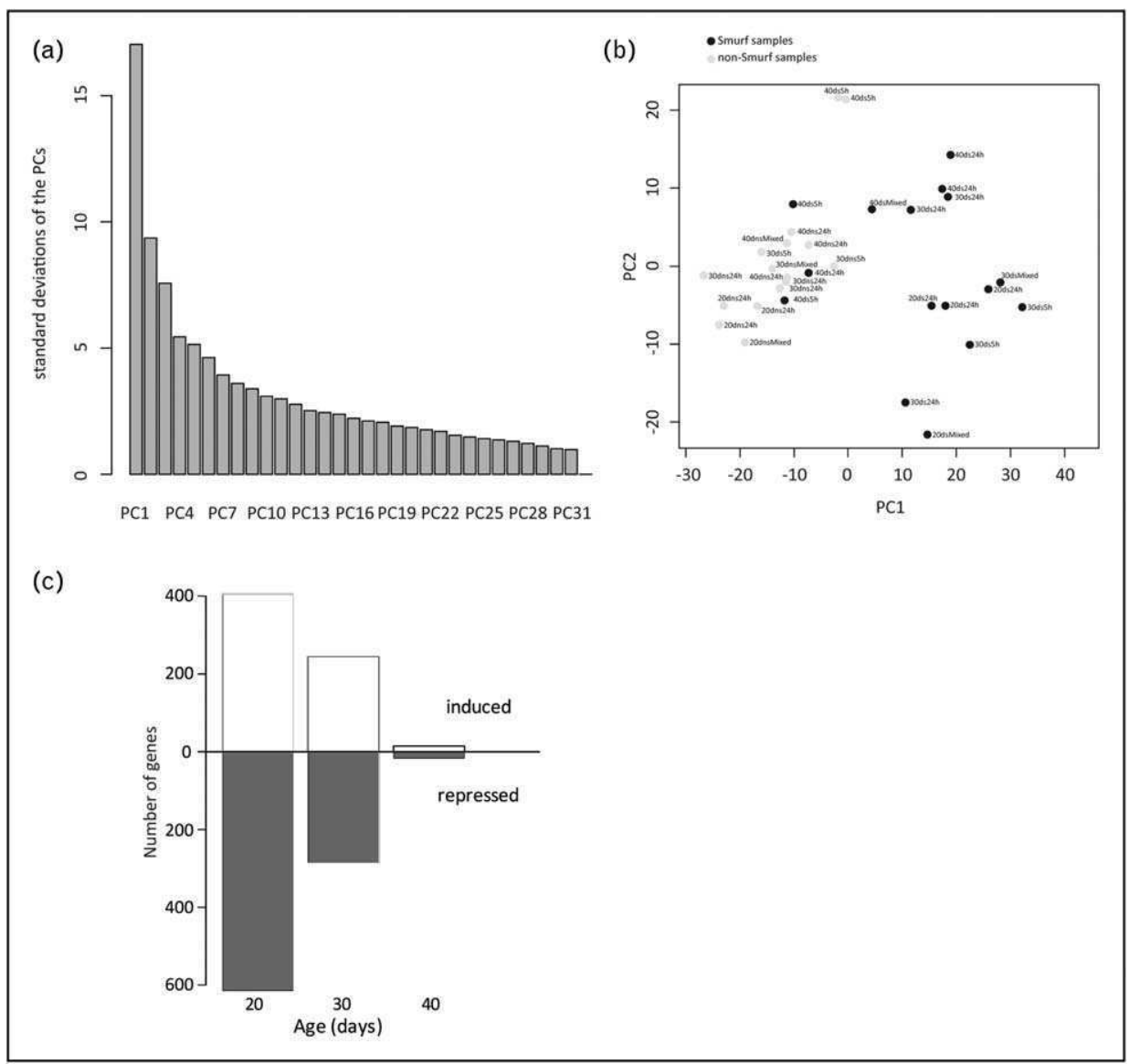

FIGURE 3. Smurf state strongly affects the transcriptom of flies. (a and b) One of the most important components obtained from a principal component analysis (PCA) of RNA samples from flies of different ages, Smurf status and time as Smurf is the Smurf state. Samples were generated from flies of 20, 30 and 40 days of age that had been assayed for Smurfness within 5, 12 or $24 \mathrm{~h}$ after removal of the mixed-age Smurf individuals. (c) The absolute number of genes differentially expressed between Smurfs and non-Smurfs decreases with age.

\section{Towards accepting the existence of two discontinuous phases of ageing in humans?}

In the past few years, a series of articles based on human studies have shown results that may be relevant to this two-phase ageing model. These studies analyse phenotypes ranging from physical abilities to molecular signatures in ageing humans.

Although data associated with physical abilities are difficult to separate from confounding factors such as accidents owing to functional impairments, the advantage of such measurements is their cost-efficiency and relatively low technical requirements for acquisition. In 2010, a systematic quantitative review of the literature was published, studying associations between objective measures of physical capability (grip strength, walking speed, chair rising and standing balance times) and mortality in community dwelling populations [23]. This meta-analysis showed that grip strength (hazard ratio $=1.17$, IC95 $=1.10-1.25$ ), walking speed (hazard ratio $=2.87$, IC95 $=2.22-3.72$ ) and chair raise speed (hazard ratio $=1.96$,
IC95 $=1.56-2.45)$ were significant predictors of the mortality risk. Interestingly, this meta-analysis identifies associations with all-causes mortality. In 2014, a PLoS One article [24] linked an altered sense of smell to a higher risk of 5-year mortality for all mortality causes (hazard ratio $=3.37$, IC95 $=2.04-5.57$ ). Strikingly, it has recently been shown that it is possible to predict among twins which one will die first using face-based age estimates [25].

More recently, much effort has been put into identifying relevant molecular markers of physiological age, thanks especially to the use of large human cohorts. A large biomarker profiling using nuclear magnetic resonance spectroscopy based on 17345 persons [26"'] revealed four circulating biomarkers $(\alpha-1$-acid glycoprotein hazard ratio $=1.67$, IC95 $=1.53-1.82$, albumin hazard ratio $=0.7$, IC95 $=0.65-0.76$, vLDL particule size hazard ratio $=0.69$, IC95 $=0.62-0.77$ and citrate hazard ratio $=1.33$, IC95 $=1.21-1.45$ ) strongly associated with a high risk of death from cancer, 


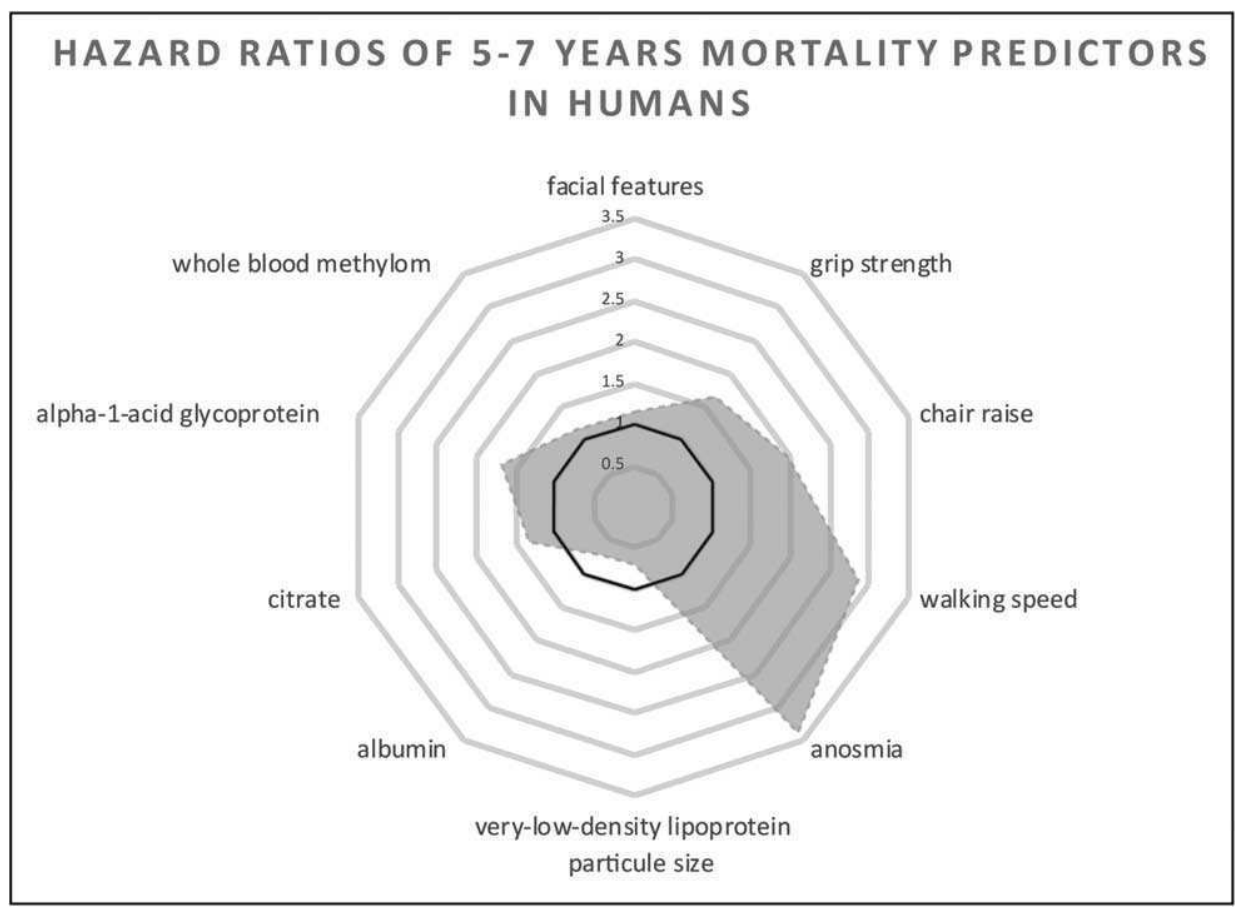

FIGURE 4. Summary of the 5-7 mortality predictors reviewed here. The indicated values are hazard ratios.

cardiovascular and noncardiovascular diseases, as well as of all-cause mortality. Inflammasome gene expression allows the stratification of 85 +year-old humans into two extreme states echoing the Smurf phenotype [27"].

Another interesting target for such physiological age markers is an epigenetic mark, the DNA methylation. Indeed, this mark plays a direct role in regulating gene expression regulation and has been shown to be affected by age in humans $[28,29]$ as well as in C. elegans [30] where transcriptional drift - the age-related dysregulation of gene expressions - rescue has been shown to postpone the onset of mortality [31]. In addition, this 'epigenetic clock' was shown to predict all-cause mortality from whole blood in the elderly [32]. This methylome clock maintains its predictive properties when studied in different ethnic contexts and sexes [33].

Although these findings strongly suggest the existence of markers of physiological ageing which may be used to predict remaining lifespan, it is not yet clear whether these markers behave like the Smurf phenotype in flies. Indeed, in drosophila, there is a sharp transition between the two states we described, with multiple features occurring concomitantly. Whether such a sharp transition occurs in humans will require longitudinal studies of wellcontrolled human cohorts, based on the initial results from previous studies.

It is interesting to note that the predictive power of integrated phenotypes is stronger than that of molecular phenotypes (Fig. 4). Although this might be due to technical differences associated with measurement noise and age of participants, it could be a sign that these integrated phenotypes occur much closer to death, thus naturally decreasing the uncertainty of the statistical association.

\section{Predicting high risk of impending death in medicine and new ethical issues}

Although these results allow us to identify strong predictors of impending death from natural causes, these studies are mostly correlative and do not bring new information about the underlying causes. Nevertheless, these markers allow for the identification of two subpopulations, each characterized by a significantly different risk of impending death from all causes. This, taken together with the fact that the currently identified, apparently unrelated markers correlate with a high 5-year mortality risk, lends credence to the hypothesis of programmed ageing in the late phase of life. This notion of a programmed ageing is still strongly debated [34"]. If so, apparently unconnected age-related diseases might simply be symptoms of this hidden programme.

Taking this into account will enable the proper study and identification of this programme in human beings. If such a programme exists and if previously discussed markers of physiological age as well as those still to discover - are measurable symptoms of this programme, then we can identify 
years in advance which part of the population is at higher risk of death. This would then allow to study the chain of events occurring in these individuals as well as design personalized care. Perhaps, this last phase of life is not curable. If so, identifying this phenotype may lead to reconsider current intervention strategies and to develop integrated, evidence based-programs for accompanying these end-oflife processes.

Indeed, between 63 and $87 \%$ of people 65 years old and above show a chronic condition [35]. The high healthcare cost of individuals in their last year of life, and/or affected by chronic diseases, is under scrutiny as estimates show that 'for the population aged 65 and over in 2002, 5\% of patients in the last year of life generated approximately half the hospital expenditures for that age group' [36]. On the contrary, the palliative approach to treating end-oflife patients is shown to have beneficial effects for the patient and their family [37], with even an improvement of short-term survival compared to classic care [38].

Such diagnostic capabilities would come at a price. The ability to identify, a few years in advance - with large margin of error - whether a patient is at high risk of death would raise new important ethical issues: Would it be judicious to offer patients such analysis for better-adapted therapeutic options? How would the results be communicated to the patient? How would healthcare policy evolve under this new paradigm given the aforementioned economic concerns? How would it impact personal finances, such as health insurance and loan attributions? Undoubtedly, this will require a large public debate. Furthermore, we may expect strong demand for pharmacological treatments or preventive behavioural changes that would delay the onset of the latter phase of life. This implies a large effort in fundamental research to better understand the mechanisms involved in this transition. The use of model organisms with powerful genetic tools and already available theoretical frameworks will be invaluable in reaching this goal.

\section{CONCLUSION}

The link between multiple physiological and molecular phenotypes and impending death in a shortlived organism was a fortunate discovery. For longer lived organisms such as humans, the chain of events might be stretched in time, thus making it less obvious to connect the dots between multiple age-related phenotypes and impending death. If the research and medical communities take a common approach to the problem of ageing and agerelated diseases, we might be able to identify early events characterizing a subpopulation at risk of diseases and death from all causes in humans. Whether or not some of the chronic diseases that we try to treat today are only the phenotypes associated with a chronic state, somehow programmed, which precedes death, warrant an investigation. This may change the way we treat these patients, probably allowing for a greater allocation of health budgets towards palliative care for individuals diagnosed as being in the 'predeath' phase. This will modify our perception of ageing itself, as by becoming more predictable it will become less startling. Of course, being able to predict death raises deep ethical, legal and societal questions that will have to be addressed thoroughly in the coming years.

\section{Acknowledgements}

We thank Dr Anahi Molla-Herman, Dr Clément Carré and Dr Frédéric Bernard for their useful comment on the article as well as Elizabeth Howard for her comments and language editing.

\section{Financial support and sponsorship} CNRS.

\section{Conflicts of interest}

There are no conflicts of interest.

\section{REFERENCES AND RECOMMENDED}

\section{READING}

Papers of particular interest, published within the annual period of review, have been highlighted as:

- of special interest

- of outstanding interest

1. Jones $O R$, Scheuerlein $A$, Salguero-Gómez $R$, et al. Diversity of ageing across - the tree of life. Nature 2014;505:169-173.

This article reviews the different survival, mortality and fertility profiles existing in living organisms.

2. Kirkwood TB, Austad SN. Why do we age? Nature 2000; 408:233-238.

3. Lopez-Otin C, Blasco MA, Partridge L, et al. The hallmarks of aging. Cell 2013; 153:1194-1217.

4. Landis GN, Abdueva D, Skvortsov D, et al. Similar gene expression patterns characterize aging and oxidative stress in Drosophila melanogaster. Proc Natl Acad Sci U S A 2004; 101:7663-7668.

5. Girardot F, Lasbleiz C, Monnier V, Tricoire H. Specific age-related signatures in Drosophila body parts transcriptome. BMC Genomics 2006; 7:69.

6. Pletcher SD, Macdonald SJ, Marguerie R, et al. Genome-wide transcript profiles in aging and calorically restricted Drosophila melanogaster. Curr Biol 2002; $12: 712-723$.

7. Rea SL, Wu D, Cypser JR, et al. A stress-sensitive reporter predicts longevity in isogenic populations of Caenorhabditis elegans. Nat Genet 2005; 37: 894-898.

8. Gerstbrein B, Stamatas G, Kollias N, Driscoll M. In vivo spectrofluorimetry reveals endogenous biomarkers that report healthspan and dietary restriction in Caenorhabditis elegans. Aging Cell 2005; 4:127-137.

9. Mueller LD, Shahrestani $P$, Rauser CL. Predicting death in female Drosophila. Exp Gerontol 2009; 44:766-772.

10. Mueller LD, Shahrestani $P$, Rauser $C L$, Rose MR. The death spiral: predicting death in Drosophila cohorts. Biogerontology 2016; 17:805-816.

11. Rera $M$, Bahadorani $S$, Cho J, et al. Modulation of longevity and tissue homeostasis by the Drosophila PGC-1 homolog. Cell Metab 2011; 14:623-634.

12. Rera M, Clark RI, Walker DW. Intestinal barrier dysfunction links metabolic and inflammatory markers of aging to death in Drosophila. Proc Natl Acad Sci U S A 2012; 109:21528-21533. 
13. Ren $C$, Webster $P$, Finkel SE, Tower J. Increased internal and external bacterial load during Drosophila aging without life-span trade-off. Cell Metab 2007; 6:144-152.

14. Minois N, Khazaeli AA, Curtsinger JW. Locomotor activity as a function of age and life span in Drosophila melanogaster overexpressing hsp70. Exp Gerontol 2001; 36:1137-1153.

15. Tricoire $H$, Rera $M$, New A. Discontinuous 2 phases of aging model: lessons

-1 from Drosophila melanogaster. PLoS One 2015; 10:e0141920.

A mathematical model of ageing based on the Smurf phenotype, which introduces three parameters easily measurable experimentally.

16. Dambroise $E$, Monnier $L$, Ruisheng $L$, et al. Two phases of aging separated by

- the Smurf transition as a public path to death. Sci Rep [Internet] 2016; 6:23523; Sep [cited 2017 Aug 17];6(1). Available from: http://www.nature.com/articles/srep23523.

Describes an extension of the Smurf phenotype to other model organisms including nematodes and zebrafish.

17. Tran L, Greenwood-Van Meerveld B. Age-associated remodeling of the intestinal epithelial barrier. J Gerontol Biol Sci Med Sci 2013; 68: 1045-1056.

18. Ma SW, Foster DO. Potentiation of in vivo thermogenesis in rat brown adipose tissue by stimulation of alpha 1-adrenoreceptors is associated with increased release of cyclic AMP. Can J Physiol Pharmacol 1984; 62: 943-948.

19. Harris CE, Griffiths RD, Freestone $N$, et al. Intestinal permeability in the critically ill. Intensive Care Med 1992; 18:38-41.

20. Driver C. The Gompertz function does not measure ageing. Biogerontology $2001 ; 2: 61-65$.

21. Ricklefs RE, Scheuerlein A. Biological implications of the Weibull and Gompertz models of aging. J Gerontol Biol Sci Med Sci 2002; 57: B69-B76.

22. Wilson DL. The analysis of survival (mortality) data: fitting Gompertz, Weibull, and logistic functions. Mech Ageing Dev 1994; 74:15-33.

23. Cooper R, Kuh D, Hardy R; Mortality Review Group, FALCon and HALCyon Study Teams. Objectively measured physical capability levels and mortality: systematic review and meta-analysis. BMJ 2010; 341:c4467.

24. Pinto JM, Wroblewski KE, Kern DW, et al. Olfactory dysfunction predicts 5year mortality in older adults. PLoS One 2014; 9:e107541.

25. Gunn DA, Larsen LA, Lall JS, et al. Mortality is written on the face. J Gerontol A Biol Sci Med Sci 2016; 71:72-77.
26. Fischer $\mathrm{K}$, Kettunen J, Wurtz $\mathrm{P}$, et al. Biomarker profiling by nuclear magnetic

- resonance spectroscopy for the prediction of all-cause mortality: an observational study of 17,345 persons. PLoS Med 2014; 11:e1001606.

Identification of biomarkers predicting all-cause mortality in humans.

27. Furman D, Chang J, Lartigue L, et al. Expression of specific inflammasome

- gene modules stratifies older individuals into two extreme clinical and immunological states. Nat Med 2017; 23:174-184.

Work echoing the Smurf status in $85+$ year-old humans based on inflammation genes expression.

28. Bell JT, Tsai P-C, Yang T-P, et al. Epigenome-wide scans identify differentially methylated regions for age and age-related phenotypes in a healthy ageing population. PLoS Genet 2012; 8:e1002629.

29. Bollati V, Schwartz J, Wright R, et al. Decline in genomic DNA methylation through aging in a cohort of elderly subjects. Mech Ageing Dev 2009; 130:234-239.

30. Pincus Z, Slack FJ. Transcriptional (dys)regulation and aging in Caenorhabditis elegans. Genome Biol 2008; 9:233.

31. Rangaraju S, Solis GM, Thompson RC, et al. Suppression of transcriptional drift extends $\mathrm{C}$. elegans lifespan by postponing the onset of mortality. eLife 2015; 4:e08833.

32. Marioni RE, Shah $\mathrm{S}, \mathrm{McR}$ ae $\mathrm{AF}$, et al. DNA methylation age of blood predicts all-cause mortality in later life. Genome Biol 2015; 16:25.

33. Horvath $\mathrm{S}$, Gurven $\mathrm{M}$, Levine $\mathrm{ME}$, et al. An epigenetic clock analysis of race/ ethnicity, sex, and coronary heart disease. Genome Biol [Internet] 2016; 17:171; Aug 11;17. Available from: http://www.ncbi.nlm.nih.gov/pmc/articles/PMC4980791/

34. Kowald A, Kirkwood TBL. Can aging be programmed? A critical literature

- review. Aging Cell 2016; 15:986-998

An interesting discussion about arguments for and against a programmed ageing theory.

35. Aldridge MD, Kelley AS. The myth regarding the high cost of end-of-life care. Am J Public Health 2015; 105:2411-2415.

36. Seshamani M, Gray A. Time to death and health expenditure: an improved model for the impact of demographic change on healthcare costs. Age Ageing 2004; 33:556-561.

37. Kaur J, Mohanti BK. Transition from curative to palliative care in cancer. Indian J Palliat Care 2011; 17:1-5.

38. Temel JS, Greer JA, Muzikansky A, et al. Early palliative care for patients with metastatic non-small-cell lung cancer. N Engl J Med 2010; 363:733-742. 